\title{
L'apport des correspondants genevois de Spallanzani à la méthodologie expérimentale *
}

par Mirko D. Grmek

RéSUMÉ

Certains traits de l'œuvre de Lazzaro Spallanzani correspondent aux valeurs principales de la haute bourgeoisie genevoise de son temps. C'est auprès de Charles Bonnet, de Jean Senebier et d'Abraham Trembley que le naturaliste italien a trouvé le meilleur accueil et la plus grande compréhension. La correspondance entre ces savants est une source particulièrement riche pour la reconstruction des fondements épistémologiques de la méthodologie expérimentale dans les sciences de la vie pendant la seconde moitié du XVIII e siècle. L'Essai sur l'art d'observer et de faire des expériences du pasteur Senebier est une analyse magistrale et une sorte de long sermon en faveur d'une méthode de recherche que l'abbé Spallanzani ne prêchait que par l'exemple.

Le choix du titre de cette conférence s'est imposé d'emblée par la convergence de considérations de trois ordres: l'importance et le caractère transdisciplinaire du sujet, son lien avec Genève et, enfin, la possibilité d'une analyse historique renouvelée grâce à la publication récente de certaines sources.

Peu d'aspects de la mentalité moderne sont aussi significatifs que la valeur accordée à la méthode expérimentale. Et pourtant les historiens ne lui prêtent que trop rarement l'attention qu'elle mérite.

En visitant les anciens appartements de Louis Pasteur à Paris, transformés aujourd'hui en musée, on peut constater que, très attaché aux symboles, ce savant avait mis dans sa salle à manger deux portraits grandeur nature: le sien et celui de Lazzaro Spallanzani, naturaliste qu'il tenait pour son grand prédécesseur. D'autres expérimentateurs illustres ont souligné leur dette envers Spallanzani et quelques historiens des sciences, par exemple Jean Rostand, n'ont pas hésité à voir en lui «le fondateur de la biologie expérimentale».

Fort curieusement, ce savant italien, professeur et de surcroît prêtre catholique, a trouvé de son vivant les esprits les plus conformes au sien parmi les érudits d'un pays étranger, de religion protestante et dépourvus du

* Conférence Markus Guggenheim-Schnurr 
prestige que confèrent les charges universitaires. En effet, c'est auprès de Charles Bonnet, de Jean Senebier, d'Abraham Trembley et de quelques autres patriciens genevois que l'abbé Spallanzani, observateur minutieux, rationaliste impénitent et homme de vérité, a trouvé le meilleur accueil et la plus grande compréhension.

Au-delà des sympathies personnelles, une affinité idéologique profonde rapprochait Spallanzani des naturalistes mentionnés. Certains traits de son œuvre correspondaient de façon idéale à ce qu'étaient, selon les analyses sociologiques d'Alphonse de Candolle, d'André Sayous et de Cléopâtre Montadon, les valeurs principales de la haute bourgeoisie genevoise du $\mathrm{XVIII}^{\mathrm{e}}$ siècle, à savoir la quête intransigeante de la vérité, l'éthique du travail, le sens du devoir et la minutie dans l'exécution des buts poursuivis. Ils voulaient augmenter par un effort incessant le corpus des connaissances sûres, produire une sorte de «capital scientifique».

La méthode de Spallanzani est tout d'abord celle du bon sens. Le lecteur de ses écrits l'assimile sans difficulté aucune. Une situation apparemment paradoxale en résulte: d'une part, cette méthode est limpide et évidente; d'autre part, elle résiste à l'analyse épistémologique. Presque tous les philosophes des sciences la passent sous silence. Pour eux, Spallanzani est le «mauvais exemple» par excellence. On ne peut dans son raisonnement expérimental distinguer le contexte de la découverte de celui de la justification et on ne peut réduire la réalité de ses procédés d'investigation ni à l'inductivisme baconien, ni à la méthodologie galiléenne, newtonienne ou bernardienne, ni à la philosophie de Popper ou de Lakatos.

La seule personne que Spallanzani, homme introverti et fier, reconnaissait publiquement comme son maître et dont il sollicitait les conseils était Charles Bonnet. Et ce n'est pas pour faire un vain compliment que, après onze ans de relations amicales, il lui écrit humblement: «Entre vous et moi il y aura toujours la différence qui passe entre le maître et l'écolier». ${ }^{1}$

Les relations épistolaires entre Spallanzani et Bonnet ont commencé à l'initiative du naturaliste italien. Au moment où, le 18 juillet 1765, Spallanzani adresse à Bonnet sa première lettre, ce dernier est déjà une sommité internationalement reconnue. Spallanzani, son cadet de neuf ans, n'est encore qu'au début de sa carrière scientifique. Le premier contact intervient par l'intermédiaire de Vallisneri, professeur à Padoue, qui transmet à Bonnet l'ouvrage de Spallanzani comportant la réfutation des expériences de Needham sur la génération spontanée des infusoires. 
Bonnet renvoie la politesse en expédiant à Spallanzani son ouvrage le plus récent. Dès sa toute première lettre au savant italien, datée de Genève le 14 septembre 1765 , il insiste sur la valeur méthodologique de ses propres livres.

«J'ai été très flatté - écrit Bonnet - de l'approbation que vous avez bien voulu donner à mes Considérations sur les Corps organisés. Je serois bien compensé de mon travail, si ce livre devenoit une Logique à l'usage du naturaliste... J'ai mis au jour un autre ouvrage, sous le titre Contemplation de la Nature... Arrêtez-vous surtout à la préface et aux trois chapitres de la partie VIII : c'est encore là un essai de Logique, que j'adresse aux jeunes naturalistes.» ${ }^{2}$

«Oui - répond Spallanzani-je crois qu'il n'y ait livre plus propre pour former un naturaliste que le vôtre. Il enseigne l'art de penser, et tout à la fois celui d'expérimenter; et l'un et l'autre y est insinué d'une délicatesse qui touche au dernier degré. ...Je m'efforcerai dans l'avenir d'être moins insensible qu'il me sera possible à vos sçavantes instructions. Je me prendrai aussi hardiesse, comme je le fais à présent de recourir à vos conseils en fait d'histoire naturelle.» ${ }^{3}$

Bonnet, ravi de cette demande, conseille à Spallanzani de reprendre les expériences de Réaumur sur la régénération des pattes de l'écrevisse. Dans une longue lettre, il énumère une série de sujets dignes d'être étudiés par la méthode expérimentale mais termine ce panorama de desiderata par une mise en garde:

«Ne multipliez pas trop les objets de vos recherches: je préférerois que vous n'en suivissiez que deux ou trois; l'attention, ce puissant ressort, en seroit moins partagée, et vous verriez mieux et plus à fond dans votre objet.» ${ }^{4}$

Pendant 26 ans, Spallanzani et Bonnet ont échangé environ 200 lettres, souvent très longues et presque toujours pleines d'idées originales et d'informations peu banales. Bonnet s'efforce d'orienter Spallanzani vers la confirmation expérimentale de la préformation des germes. Après avoir suggéré des expériences astucieuses sur la régénération, il le pousse à tenter des fécondations artificielles. Ainsi, Bonnet écrit le 8 août 1767: «Il me vient une idée : ne pourriez-vous point essayer de féconder des œufs de grenouilles sans l'intervention du mâle? Je m'explique: il faudroit tenter d'extraire des vésicules du mâle la liqueur prolifique et d'en arroser un certain nombre d'œufs. Il faudrait encore tenter de répandre cette même liqueur sur des œufs de genres et d'espèces différents.» ${ }^{5}$ On connaît, si j'ose m'exprimer ainsi, la fécondité extraordinaire de cette idée.

Dans une lettre à Albrecht von Haller, Bonnet caractérise de la façon suivante le début de sa collaboration avec Spallanzani: 
«En me prévenant de la manière la plus obligeante l'Abbé Spallanzani me demanda quelques directions pour continuer à parcourir la carrière où il venait d'entrer... Je lui indiquai ce que j'aurois fait moi même si mes yeux me l'avoient permis. Je l'adressai surtout aux reproductions animales, comme à une source intarissable de vérités nouvelles... Privé moi même actuellement du plaisir de servir le public en qualité d'observateur, il me sembloit que je mettois un homme à ma place, et que je contemplois encore la nature par de nouvelles lunettes». ${ }^{6}$

Le fait est que Bonnet souffrait d'une mauvaise vue et qu'il était, dès son adolescence, dur d'oreille. Dans son autobiographie, il dit que sa vie «a été presque aussi uniforme que le cours des astres» et que, à part l'excitation lors de la découverte de la parthénogenèse des pucerons et d'autres aventures de l'esprit il se l'est coulée douce dans la ville de Genève, puis dans le site enchanteur de Genthod, mais qu'il a subi aussi la terrible épreuve de l'infirmité des deux sens les plus précieux.

A plusieurs occasions, Bonnet déclare que l'usage abusif du microscope l'a rendu presque aveugle et qu'il compte sur Spallanzani pour être en quelque sorte «ses yeux». ${ }^{7}$

Toutefois, contrairement à ce qu'il pensait lui-même et à ce que répètent ses biographes, Bonnet n'a pas souffert des yeux parce qu'il a fait des observations microscopiques mais il a été attiré par les observations des êtres microscopiques parce qu'il souffrait des yeux. Quant à l'usage des yeux de Spallanzani comme substitut des siens, Bonnet se berçait d'illusions. Il ressort clairement de la correspondance entre ces deux hommes que Spallanzani n'était pas un simple exécutant mais qu'il pensait avec sa propre tête. S'il prenait en considération tout ce que Bonnet lui écrivait, il ne réalisait que les expériences conformes à son propre goût.

Quel était l'enseignement méthodologique explicite de Bonnet? Dans sa correspondance, il renvoie à ce propos en particulier à la préface et à la $\mathrm{VIII}^{\mathrm{e}}$ partie de sa Contemplation de la nature. Spallanzani a lu et médité ce livre; il l'a traduit en italien, il a enrichi sa traduction de nombreuses notes personnelles et il s'en est servi dans son enseignement. Le livre de Bonnet contient effectivement quelques réflexions d'ordre méthodologique. L'auteur y souligne la priorité de l'observation sur le raisonnement, prêche l'humilité devant l'immensité des secrets de la nature, critique le recours aux analogies et conseille la méfiance à l'égard des préjugés. Toutefois, il s'agit de remarques assez banales que Spallanzani tient pour des vérités tellement évidentes qu'elles ne nécessitent aucun commentaire.

Dans ses lettres, Bonnet met Spallanzani en garde aussi bien contre une 
érudition excessive que contre l'usage précipité des analogies et des généralisations théoriques. Il dit par exemple ceci de son ami Haller:

«Son érudition est prodigieuse, mais je ne sçai si elle n'a point nui à son génie. S'il avoit moins consulté les auteurs, il auroit plus consulté sa tête, il auroit plus médité et plus découvert encore.» ${ }^{8}$

L'érudition sert peu, car «nous n'expérimentons que depuis hier», s'amuse à dire Bonnet en citant malicieusement Leibniz, philosophe qui, dit-il, «n'expérimentoit guères, mais qui méditoit sans cesse». ${ }^{9}$

\section{Selon Bonnet,}

«ce sera toujours à l'expérience à vérifier ou à détruire, mais nos conjectures peuvent conduire à des expériences instructives». ${ }^{10}$

Les hypothèses sont précieuses: elles sont inévitables aussi bien pour orienter les expériences que pour les interpréter. Bonnet le dit avec force au début de l'un de ses principaux ouvrages:

«Bannir entièrement de la Physique l'Art de conjecturer, ce seroit nous réduire aux pures Observations, et à quoi nous serviroient les Observations, si nous n'en tirions pas la moindre Conséquence? Nous amasserions sans cesse des matériaux pour ne bâtir jamais. Nous confondrions sans cesse le moyen avec la fin». ${ }^{11}$

Dans ses Considérations sur les corps organisés, Bonnet dit fièrement:

«Je n'ai tiré des faits que les conséquences qui me sembloient en découler le plus naturellement. J'ai souhaité que mon Livre fût une espèce de Logique. Je n'ai done pas mis les conjectures à la place des faits, mais j'ai fait en sorte qu'elles résultassent des faits comme de leurs principes.» ${ }^{12}$

Il revient à la charge dans une lettre à Spallanzani :

«J'ai été surtout très attentif à ne tirer des faits que les conséquences les plus immédiates. Vous le ferez aussi, j'en suis très sûr: vous possédez la Logique de l'observateur.» ${ }^{13}$

Cette conviction de Bonnet, certainement très sincère, prête à sourire. S'il a raison quant à la prudence de Spallanzani, il se trompe complètement quant à sa propre manière d'agir. Loin de pouvoir freiner son imagination à la formulation des «conséquences les plus immédiates», Bonnet se lançait à cœur joie dans l'élaboration des théories. Le naturaliste genevois reprochait à presque tous les savants de son temps de raisonner trop, ce qui ne l'empêchait nullement de remplir ses propres ouvrages d'élucubrations 
théoriques particulièrement longues et spécieuses. Tout se passait comme si Bonnet se réservait le droit de philosopher sur les propriétés générales des êtres vivants, les autres naturalistes devant se contenter de lui fournir des «faits».

Les principes de son épistémologie, Bonnet voulait les prêcher en premier lieu par son propre exemple, mais il caressait aussi le projet d'une sorte de manuel du parfait naturaliste observateur. Plusieurs années avant le début de ses relations avec Spallanzani, Bonnet écrivait déjà à Haller:

«J'ai souvent eu dans l'esprit le plan d'un Ouvrage que j'aurois intitulé Essai sur l'Art d'observer... J'aurois montré les routes par lesquelles les Grand Maîtres de l'Art sont parvenus dans le Sanctuaire de la Nature. J'aurois indiqué les obstacles qu'ils ont eu à franchir; les écueils qu'ils ont eu à éviter; les précautions qu'ils ont eu à prendre; les moyens qu'ils ont eu à employer; les différentes vues qui se sont offertes à leurs esprits; l'emploi qu'ils ont sçu en faire. J'aurois fait voir que l'esprit d'Observation est l'esprit universel des Sciences et des Arts. Mais, Monsieur, pour un Ouvrage comme celui là il me faudrait votre tête.» ${ }^{14}$

Devenu membre de la Société hollandaise des sciences, Bonnet propose en 1765 à celle-ci de mettre au concours «ce sujet si important : combien l'Art d'observer peut contribuer à la perfection de l'Esprit». ${ }^{15}$ Le concours eut effectivement lieu et se termina par le couronnement de deux ouvrages, dus à deux pasteurs suisses, Benjamin Carrard et Jean Senebier. Jamais, écrit Carrard au début de l'Essai qui remporta le premier prix, on n'avait autant observé et jamais on n'avait aussi cruellement manqué d'une méthodologie solide.

Bonnet ne fut pas satisfait du résultat: «Si j’avois jugé-écrit-il à Hallerj’aurois renvoyé le prix à deux ans, et invité les auteurs à retravailler leurs pièces, etc. Je désirois sur tout une Logique réduite en action; on nous donne une sorte d'Encyclopédie ou plutôt une sorte de Tableau encyclopédique.» ${ }^{16}$

Cette déclaration nous fait mesurer la portée du compliment que Bonnet adresse à Spallanzani en juin 1776, après avoir lu ses Opusculi : ce livre est, dit-il textuellement, «à mon avis, un des plus parfaits modèles de l'art d'observer, et une véritable Logique en action». ${ }^{17}$ Ainsi, Spallanzani aurait réalisé de manière la plus parfaite ce que Bonnet s'efforçait de faire luimême. Quant au manuel systématique de l'art d'observer, les vœux de Bonnet seront exaucés avant sa mort: ce manuel paraîtra comme un fruit tardif du concours hollandais. La collaboration de Senebier avec Spallanzani et son expérience personnelle, acquise dans un second temps, donneront une belle vigueur à un essai initialement maigre et trop livresque. 
Dans l'histoire de la méthode expérimentale, Jean Senebier entre en scène d'abord comme admirateur de Bonnet et candidat au concours hollandais, puis comme traducteur de Spallanzani, pour devenir enfin lui-même un expérimentateur avisé et l'auteur de la première synthèse des acquisitions méthodologiques du $\mathrm{XVIII}^{\mathrm{e}}$ siècle dans le domaine de l'expérimentation biologique.

Avant de parler de Senebier, je voudrais rappeler brièvement les relations qu'entretenait Spallanzani avec quelques autres savants suisses. C'est par l'intermédiaire de Bonnet qu'il échangeait des informations scientifiques avec Horace Benedict de Saussure et Abraham Trembley. ${ }^{18}$ L'été 1779. Spallanzani passa quelques jours à Genève, puis dans la maison de Bonnet à Genthod et se rendit ensuite à Berne, Bâle, Zurich et Lucerne. Bonnet fut ravi de sa visite, Spallanzani plutôt déçu du voyage dans son ensemble et désagréablement surpris de l'état selon lui assez lamentable des collections suisses d'histoire naturelle. Le 30 septembre 1779, il écrit de Reggio pour remercier Bonnet de son accueil chaleureux à Genthod.

«Je vous dirai sans détour - dit-il dans cette lettre - que de tous les pays que j'ai parcourus dans mon voyage sans en excepter la ville de Turin, Genève est la seule où j'ai trouvé de véritables naturalistes. Vous comprenez facilement que je parle de vous, de l'illustre auteur des polypes et de Mr. de Saussure.» ${ }^{19}$

Avant cette date, Spallanzani correspondait aussi, indépendamment de Bonnet, avec Haller à Berne et avec Voltaire, installé sur la frontière française, à proximité de Genève. Il admirait sincèrement le physiologiste suisse, tandis qu'il tenait l'écrivain français pour «très superficiel dans les sciences» et ne lui reconnaissait que «l'avantage des traits d'esprit». Dans une lettre à Bonnet, Spallanzani invente lui-même un «beau mot» voltairien en appelant le poète-philosophe de Ferney «une nuit pleine de vers luisans». ${ }^{20}$

Spallanzani ne connaissait pas personnellement Johann Georg Zimmermann, auteur du fameux Traité de l'expérience, et n'avait pas d'amis dans les cercles médicaux suisses, à l'exception toutefois de Samuel-Auguste Tissot, «homme le plus poli et le plus aimable du monde» avec lequel, dit Spallanzani, «j'ai avantage de converser (à Pavie) presque tous les jours, car nous habitons tous les deux la même maison». ${ }^{21}$ Assez tard dans sa vie, Spallanzani eut des contacts aussi avec Jean Louis Odier et avec Louis Jurine qui contribuera de façon décisive à la découverte de l'écholocation comme moyen d'orientation des chauves-souris. 
Bonnet poussait Spallanzani à rédiger ses travaux non pas en latin ou directement en français mais en sa langue maternelle pour les faire traduire ensuite dans la langue des encyclopédistes. Il dénicha même le traducteur idéal en la personne de Senebier. ${ }^{22}$

Le 26 juin 1776, Jean Senebier, «bibliothécaire de la République de Genève», écrit à Spallanzani :

«J'ai cru être plus utile aux sciences en mettant votre ouvrage dans une langue qui lui donnera un usage plus général, qu'en continuant de travailler aux miens.» ${ }^{23}$

Ainsi débute une relation épistolaire qui, en exprimant de plus en plus une admiration mutuelle et une amitié profonde, s'intensifie au fil des années pour se terminer seulement par la mort du naturaliste italien. Entre 1776 et 1799, Spallanzani et Senebier ont échangé environ 300 lettres. Au fur et à mesure que cette correspondance prenait de l'ampleur, la correspondance entre le naturaliste italien et Bonnet s'amenuisait. A Bonnet, Spallanzani écrit exclusivement en français, au second, le plus souvent en italien, donc avec plus de spontanéité et de chaleur. Le rapport maître-disciple est renversé.

Le contenu des lettres échangées entre Spallanzani et Senebier est très varié. Toute l'œuvre expérimentale de Spallanzani y passe. Les questions faussement naïves et les suggestions à la fois respectueuses et insistantes de Senebier sont souvent utiles, parfois même très importantes (par exemple à propos des propriétés du suc gastrique et de son usage comme antiseptique et à propos du rôle de l'oüe dans l'orientation nocturne des chauves-souris). Senebier s'étant mis lui-même à expérimenter, la discussion sur ses recherches prend peu à peu le dessus. Ainsi, une partie essentielle des lettres écrites avant la mort de Spallanzani est consacrée à la respiration des végétaux, domaine dans lequel la contribution de Senebier est fondamentale.

Dans cette correspondance, aucun naturaliste italien ou français ne trouve grâce: pour les uns, on admet qu'ils pensent correctement mais ceuxlà ne savent pas observer; pour d'autres, on reconnaît leur habileté manuelle ou leur don d'observation mais on les trouve «mauvais logiciens». Seuls les savants suisses sont traités avec quelques égards. L'un d'entre eux, Charles Bonnet, est toujours mentionné avec le plus grand respect.

En apprenant sa mort, Spallanzani adresse à Senebier, le 29 juillet 1793, une lettre émouvante où il parle aussi bien de sa dette personnelle envers le défunt que des qualités exceptionnelles de ce dernier. Selon lui, Bonnet était l'exemple unique d'un homme auquel la nature a donné aussi bien le talent 
de métaphysicien que celui de naturaliste; en outre, il savait s'incliner devant les arguments contraires à son opinion initiale. ${ }^{24}$

En glorifiant ainsi l'attitude «faillibiliste», cette capacité rare de changer d'avis, Spallanzani s'est souvenu peut-être de ses premiers pas dans la recherche scientifique et de sa propre conversion à propos de la génération spontanée. Cependant, il y a une ironie du sort dans le fait que l'influence de Bonnet, elle précisément, a empêché Spallanzani d'interpréter de façon correcte ses expériences sur le rôle des spermatozoïdes dans la fécondation. ${ }^{25}$

Senebier a traduit plusieurs ouvrages de Spallanzani. Les traités sur la digestion, sur la génération et sur la respiration, il ne les a pas seulement traduits mais il les a aussi assortis de considérations méthodologiques. Les études récentes de Carlo Castellani, fondées sur l'analyse des cahiers d'expériences et d'autres manuscrits inédits de Spallanzani, montrent que ces reconstructions épistémologiques de Senebier sont foncièrement erronées. L'érudit genevois a fait siennes les idées de Spallanzani sur le rôle de l'induction et s'est laissé berner par la présentation épurée et logiquement arrangée que Spallanzani donne du déroulement de ses découvertes. ${ }^{26}$

En 1802, Senebier publie chez le libraire Paschoud à Genève un livre intitulé Essai sur l'art d'observer et de faire des expériences. Dans l'Introduction générale, il dit avoir fait imprimer auparavant

\footnotetext{
«ce que j'appelai alors improprement l'Art d'observer; cet ouvrage fut traduit en allemand par Gmelin qui l'enrichit de notes. Enfin l'édition épuisée a engagé quelques libraires à former le projet de réimprimer cet ouvrage... Je trouvai que le titre qu'il portait était trop fastueux... et je sentais que je ne pourrais offrir qu'un Essai... Vingt-cinq ans mûrissent bien les idées, ils m'ont fait mieux connaître la mesure de mes forces, et le vrai sens des mots que j'emploie». ${ }^{27}$
}

Si le nouveau titre paraît plus modeste, le contenu de cette deuxième (en fait troisième ${ }^{28}$ ) édition est considérablement plus riche. Depuis 1775 , l'auteur a fait, «beaucoup d'observations et d'expériences nouvelles»; il a suivi d'un regard attentif «les détails de cette logique» et n'en disserte plus avec des connaissances purement littéraires. Il a profité des conseils éclairés de quelques amis philosophes et expérimentateurs.

«Spallanzani, - dit Senebier - mon ancien ami et le dépositaire de mes pensées philosophiques, et Prévost, professeur de philosophie à Genève, m'ont encouragé à reprendre le travail de mon Art d'observer, et m'ont communiqué quelques remarques sur la première édition de cet ouvrage. ${ }^{29}$ 
Lors de la parution de l'édition révisée, Spallanzani n'était plus en vie, mais ses lettres prouvent qu'il a effectivement lu et commenté la version de 1775 . Dans une lettre écrite en mai 1777, Spallanzani accuse réception du livre, souligne son utilité pédagogique et fait des compliments d'usage, mais ne s'arrête sur aucun détail. ${ }^{30}$ Senebier n'est pas dupe:

«Vous avez traité - écrit-il à Spallanzani - mon Art d'observer comme on traite le fils unique d'un père tendre lors qu'on lui parle... mais vous comprenez bien que le modèle des observateurs devoit trouver mille défauts à mon ouvrage... Permettez moi de vous le dire naïvement: depuis que j'ai étudié vos Opuscules, je sens que je ferai des changements importants à mon ouvrage.» ${ }^{31}$

Sept ans plus tard, Senebier, travaillant sur sa nouvelle édition, demande des conseils à Spallanzani. Ce dernier lui répond par une lettre en italien dans laquelle il loue le côté pédagogique de cette entreprise, suggère des ajouts concernant des découvertes récentes, notamment dans le domaine de la chimie, recommande à Senebier d'assortir les descriptions des découvertes de raisonnements expérimentaux pour mieux mettre en valeur le génie particulier de certains rares observateurs et, enfin, propose une sorte de classification des expérimentateurs. Il y en a, dit Spallanzani, qui sont très habiles commes observateurs mais qui ne savent pas tirer des conclusions; ils cumulent des matériaux mais ne construisent rien; d'autres, en revanche, construisent trop hardiment et se fondent plus sur leur imagination que sur des faits bien établis; le vrai mérite consiste à savoir équilibrer ces deux qualités en quelque sorte contradictoires. ${ }^{32}$

Senebier est ravi du fait que Spallanzani se prête à la discussion sur ce sujet:

«Vos réflexions sur l'art d'observer - écrit-il - m'enchantent... Je me propose de mettre surtout à contribution vos ouvrages et j'y ai trouvé une foule de choses oubliées ou négligées dans le mien... Oh, je le sens bien, la classe des observateurs est moins nombreuse que celle des bons poètes exquis. Quant aux autres classes, on pourroit les former par la différence que met entre les manœuvres le plus ou le moins de pénétration, d'adresse, d'attention, etc., et on termineroit cette nomenclature, comme vous, par celle des observateurs romanciers. Le tarif que vous me proposez de faire des observations et des observateurs offre quelque chose de très piquant, de très curieux, de très difficile; je m'en occupe, mais il faudroit que ce chapitre eût été fait par vous... Je vous en ai assez dit pour faire comprendre que vous serez un des héros de mon ouvrage... Encouragez vous donc, mon excellent maître, communiquez moi, comme vous les faites espérer, quelques unes de vos grandes idées sur l'art d'observer. Je ne le croirois bien fait que s'il pouvoit devenir rigoureusement votre portrait.» ${ }^{33}$

Spallanzani réagit en avril 1795 par une autre lettre en italien, très détaillée dans la partie consacrée aux expériences de Senebier sur la physiologie 
végétale et comportant un passage bref mais vigoureux sur le livre méthodologique. Cet ouvrage sera utile aux contemporains et passera à la postérité, car c'est, dit Spallanzani, un classique dans son genre, livre sur un sujet tellement vaste et difficile que personne, à la seule exception de Petrus van Musschenbroek dans un discours limité à la physique, n'a eu à la fois le courage et le talent d'affronter avec succès. ${ }^{34}$

A la fin du XVIII ${ }^{\mathrm{e}}$ siècle, les physiciens, en premier lieu Newton, étaient encore pour tous, naturalistes et médecins inclus, des maîtres paradigmatiques. Si Bonnet est cité par Senebier comme l'inspirateur principal de son traité méthodologique, les deux grands modèles sont Newton et Lavoisier. La biologie naissante se veut fille de la physique et de la chimie. Mais la plupart des exemples concrets qui illustrent les raisonnements et les préceptes de Senebier sont tirés des sciences de la vie. Bien entendu, une place d'honneur est réservée aux pucerons de Bonnet et aux polypes de Trembley, mais Senebier décrit et commente aussi les découvertes de Réaumur, Haller, Swammerdam, Malpighi, Duhamel, Huber et autres naturalistes postérieurs au milieu du $\mathrm{XVII}^{\mathrm{e}}$ siècle.

Comme il l'a promis dans sa lettre à Spallanzani, Senebier le présente comme un héros idéal, champion sans faille de la méthode expérimentale. Dans les trois volumes de l'Essai, on trouve éparpillées des informations sur les aspects les plus divers des investigations de Spallanzani : la génération des infusoires, la régénération des parties mutilées, en particulier de la tête coupée des limaçons, l'influence de la variation des conditions du milieu sur les animalcules et sur les végétaux, la vraie nature des «vers spermatiques», les particularités des graines végétales, la reproduction des anguilles, le sort des aliments dans le gésier des gallinacés et l'action du suc gastrique, la physiologie de la respiration, les observations vulcanologiques, le comportement des gouttes d'eau sur le verre fondu, etc. ${ }^{35}$ Le lecteur actuel pourrait s'étonner de l'omission des expériences de Spallanzani sur la fécondation artificielle; elles étaient, certes, sensationnelles pour l'époque, mais Senebier les trouvait sans doute scabreuses. Son livre s'adressait aux jeunes gens, le beau sexe non exclu, et devait donc respecter leur pudeur.

Au départ, le livre de Senebier concernait seulement l'art d'observer correctement la nature. La dernière édition contient une nouvelle partie intitulée «l'art de faire les expériences». Cet ajout correspond à un changement profond et subtil d'appréciation épistémologique des procédés d'investigation. 
Tout d'abord, Senebier faisait sienne la conception de Zimmermann pour qui une expérience n'était rien d'autre qu'une observation provoquée

«L'observateur - disait Zimmermann - écoute la nature; celui qui expérimente, l'interroge.» ${ }^{36}$

Ou, pour citer les propres paroles de Senebier,

«l'observation est ce regard réfléchi que l'âme porte, par le moyen des sens, sur les objets qui l'occupent, pour acquérir une connaissance exacte de leurs qualités, de leurs effets, de leurs rapports et de leurs causes», tandis que «l'expérience est une ressource pour pénétrer ce qui échappe au sens, en forçant les objets par une préparation artificielle à mettre au jour ce qu'ils cachent». ${ }^{37}$

Une définition à la fois élégante et vague lui permet de confondre les deux procédés:

«L'art d'observer - déclare-t-il - est celui d'acquérir des idées claires et exactes sur les objets qui peuvent ébranler les sens, et de les communiquer aux autres comme on les a reçues.» ${ }^{38}$

Dans la dernière édition de son ouvrage, Senebier va plus loin que Zimmermann dans la différenciation entre l'observation et l'expérience. L'observateur, dit-il, «regarde la nature comme un livre, dont il doit lire rigoureusement les caractères, sans leur imaginer une signification»; celui qui fait des expériences cherche à deviner les sens et pour cela «il force la nature à quitter son aspect ordinaire, il en fabrique une nouvelle». ${ }^{39}$

Selon Senebier, l'observation doit toujours précéder l'expérience, mais elle-même ne commence pas par un esprit vide. Il faut au départ accepter les recours à des autorités, ce qui pour notre auteur veut dire aux affirmations non pas des philosophes mais des savants peu suspects d'avoir été aveuglés par l'esprit de système. L'érudition est à la fois un obstacle pour la recherche des vérités nouvelles et une nécessité, car «pour chercher ce qu'on ignore, il faut savoir ce qu'on connaît». ${ }^{40}$ Pour bien observer la nature, il faut avoir des connaissances et il faut raisonner correctement. L'observation exige le recours constant à une sorte de logique.

«L'observation - dit Senebier - suppose le raisonnement, sans lui, on apercevrait sans remarquer, et l'on verrait sans voir; il faut donc méditer un sujet avant de commencer ses observations.» ${ }^{41}$

On passe donc de l'érudition et de la réflexion à l'observation, de celle-ci à l'expérience et, enfin, de cette interrogation de la nature à la théorie, aux lois. Le dernier pas est le plus difficile, le plus incertain. L'observation simple 
est souvent plus sûre que l'expérimentation. Ainsi, l'histoire des sciences montre que

«les expériences mal dirigées ou mal faites ont produit ces systèmes bizarres, qui ont si fort retardé les progrès de nos connaissances. L'expérience présentait à Descartes le fil de ses romans ingénieux; l'observation dictait à Newton la vérité de ses sublimes théories». ${ }^{42}$

L'investigation expérimentale suit, toujours d'après Senebier, soit la méthode analytique, soit la méthode synthétique. On expérimente en répétant les observations, en variant les facteurs, en modifiant la durée, en créant des conditions supplémentaires. Le passage du particulier au général, la naissance d'idées nouvelles, se fait grâce au génie personnel de l'expérimentateur. Senebier souligne le rôle de l'analogie et de l'induction, procédés logiques pleins d'embûches mais indispensables. L'analogie, dit-il «est dangereuse dans son emploi lorsqu'on s'en sert légèrement», mais «elle devient un instrument très utile et même indispensable, quand on s'en sert avec sagesse et avec prudence». ${ }^{43}$

Quant à l'induction, Senebier sait très bien que :

«On peut aisément se tromper en formant les lois (à partir des observations particulières); on suppose quelquefois une uniformité qui n'existe pas; on généralise trop vite les faits particuliers. Cependant, c'est l'art de bien généraliser ses idées qui fait le grand observateurn. ${ }^{44}$

«C'est une caractéristique des grands observateurs de faire seulement un petit nombre d'observations capitales sans réplique, mais fécondes en conséquences importantes.» ${ }^{45}$

Bien qu'il se réclame haut et fort de l'enseignement méthodologique de Newton, Senebier ne se rend pas vraiment compte de certaines caractéristiques essentielles de la méthode hypothético-déductive. Il comprend mieux le rôle heuristique des hypothèses en aval de l'observation qu'en amont.

Voici comment Senebier définit les étapes de cet iter mentis in veritatem qu'est pour lui l'investigation scientifique dans le domaine de l'histoire naturelle:

«L'étude de la nature se borne à observer les faits avec attention, à les soumettre au creuset de l'expérience, à les dénaturer pour en faire l'analyse, à les reproduire pour vérifier les procédés employés, à les rassembler pour trouver leurs rapports, à les classer pour réunir ceux qui sont analogues, à graduer leur importance pour remarquer ceux qui sont la clef des autres, à les lier entr'eux, et à saisir enfin l'expression des phénomènes que la nature place sous ses sens. ${ }^{46}$

Ce résumé magistral de la méthodologie d'un groupe de naturalistes ne doit pas faire croire que le livre de Senebier soit très abstrait et, somme toute, 
ennuyeux et pédant. Malgré un style assez pompeux, propre aux sermons de l'époque, on peut encore aujourd'hui le lire avec un véritable plaisir. C'est en fait une sorte d'histoire des principales découvertes biologiques du XVIII ${ }^{\mathrm{e}}$ siècle racontée d'une manière originale. Les raisonnements y tiennent relativement peu de place et font parfois appel à des métaphores amusantes.

Le but de ce livre est d'éveiller l'intérêt et de faire naître l'amour pour une activité intellectuelle que Senebier tient pour particulièrement noble et utile. «Je ne me dissimule pas - écrit-il - que cet ouvrage ne fera pas plus d'observateurs que les poétiques ne font de poètes.» ${ }^{47}$ Ce qui fait un grand expérimentateur, ce n'est ni l'érudition, ni la perfection des sens, ni la connaissance des méthodes d'investigation mais la possession d'une puissante «force d'imagination» et la capacité de soumettre les produits de celleci à «une logique sévère». ${ }^{48}$

«Il y a - déclare Senebier avec une pointe de jalousie mélancolique - des génies heureux comme Spallanzani, qui semblent avoir un sens particulier pour découvrir la vérité, et arriver à elle en suivant la ligne droite; tandis que le plus grand nombre décrit autour d'elle une longue spirale, sans être assuré d'arriver au but.» ${ }^{49}$ 
1 Di Pietro, Carteggi, II, p.284.

2 Ibid., II, p. 8-9.

3 Lettre du 18 novembre 1765 ; ibid., II, p. 10

4 Lettre du 27 décembre 1765; ibid., II, p. 12-14.

5 Ibid., II, p. 67.

6 Sonntag, 1983, p. 751-752. Voir aussi ce que Bonnet en dit dans ses Mémoires autobiographiques, édit. Savioz, 1948, p.355-356.

7 Voir par exemple la lettre du 9 octobre 1766; Carteggi, II, p. 43.

8 Ibid., II, p.51. Cf. aussi Savioz, 1948, p. 193.

9 Carteggi, II, p. 323.

10 Ibid., II, p. 79.

11 Bonnet, Palingénésie, 1770, I, p. 73-74; cf. aussi Sonntag, 1983, p. 1153-1154.

12 Considérations, 1762, Préface, p. 19.

13 Carteggi, II, p. 72.

14 Lettre du 22 juillet 1757; Sonntag, 1983, p. 107.

15 Cf. Marx, 1974, p. 203.

16 Sonntag, 1983, p. 1039.

17 Carteggi, II, p.281. Voir aussi la lettre de Bonnet à Haller du 17 octobre 1777; Sonntag, p. 1296.

18 Voir l'éloge de Trembley dans la lettre de Spallanzani du 14 août 1784; Carteggi, II, 477.

19 Ibid., II, p. 346.

20 Lettre du 15 juillet 1768 ; ibid., $I I, p .84$.

21 Carteggi, II, p. 417.

22 Voir notamment la lettre de Bonnet à Spallanzani du 29 juin 1776; ibid., II, p. 281.

23 Ibid., VIII, p. 7.

24 Ibid., VIII, p.294.

25 Cf. Grmek, 1982, p. 346-347.

26 Voir par exemple Castellani, 1983, p. 110-111.

27 Essai, 1802, I, p. 10-11.

28 Une première version a été publiée dans les Mémoires de la Société hollandaise des sciences.

29 Essai, I, p. 14.

30 Ibid., VIII, p. 48 et 53.

31 Ibid., VIII, p. 54.

32 Lettre du 29 janvier 1795; ibid., VIII, p. 341-342.

33 Ibid., VIII, p. 353.

34 Ibid., VIII, p. 357. Dans sa correspondance, Spallanzani mentionne à plusieurs occasions la valeur des préceptes donnés par P. van Musschenbroek dans son discours De methodo instituendi experimenta physica.

35 Voir notamment Essai, 1802, t. I, p. 166, 231, 378, 379, 395, 399, 421; t.II, p.6, 10, 283, 315,324 ; t.III, p. 4, 19, 24, 28, 35, 39, 49, 54, 56, 62, 65, 66, 84, 158.

36 Traité, 1774, p.44.

37 Essai, I, 24-25.

38 Ibid., III, p.241. 
$40 \quad$ Ibid., I, 54.

41 Ibid., I, p. 147.

42 Ibid., I, 29.

43 Ibid., III, p. 272.

44 Ibid., III, p. 273.

45 Ibid., III, p. 161.

46 Ibid., I, p. 34-35.

47 Ibid., I, p. 12.

48 Ibid., III, p. 150.

49 Ibid., I, p. 143.

\section{Bibliographie}

Aloisi, Massimo, Aspetti della vita delle scienze biologiche nel XVIII ${ }^{\circ}$ secolo attraverso le lettere di L. Spallanzani a Ch. Bonnet, in Rass. Stor. Univ. Modena 7, 1977, p. 123-144.

Bay, J. Christian, Jean Senebier, 1742-1809, in Plant. Physiol. 6, 1931, p. 189-193.

Bonnet, Charles, Considérations sur les corps organisés, Amsterdam, Rey, 1762, 2 vols., Nouvelle édition, revue par F. Markovits et S. Bienaymé, Paris, Fayard, 1985.

Bonnet, La contemplation de la nature, Amsterdam, Rey, 1764, 2 vols. - Nouvelle édition révisée: Lausanne, 1770.

Bonnet, Contemplazione della natura. Opera tradotta in italiano e corredata di note dall'Abate Spallanzani, Modena, Montanari, 1769-1770, 2 vols.

Bonnet, Contemplazione della natura. Con tutte le aggiunte fatte dall'autore all'ultima edizione francese 1781, ed arricchita dalle molte note ed osservazioni fatte all'ultima veneta edizione dai signori Lazzaro Spallanzani e Francesco Ferrara, Venezia, Rosa, 1797, 4 vols.

Bonnet, La palingénésie philosophique, Genève, Philibert et Chirol, 1770, 2 vol.

Bonnet, CEuvres d'histoire naturelle et de philosophie, Neuchâtel, Fauche, 1779-1783, 18 vols.

Candolle, Alphonse de, Histoire des sciences et des savants depuis deux siècles (1873), Paris, Fayard, 1987.

Carozzi, Marguerite, Bonnet, Spallanzani and Voltaire on regeneration of heads in snails; a continuation of the spontaneous generation debate, in Gesnerus, 42, 1985, p. 265-288.

Carrard, Benjamin, Essai qui a remporté le prix de la Société hollandaise des sciences de Haarlem de 1770 sur cette question: Qu'est-ce qui est requis dans l'art d'observer, et jusqu'où cet art contribuet-il à perfectionner l'entendement?, Amsterdam, Rey, 1777.

Castellani, Carlo, Lettres de Charles Bonnet à M.L'abbé Spallanzani. Edizione critica condotta sugli originali, con introduzione e note, Milano, Episteme, 1971.

Castellani, Ontogenesi di una dissertazione: «Le osservazioni e sperienze sui vermicelli spermatici» di Spallanzani L., in Hist. Phil. Life Sci. 4, 1982, p. 125-157.

Castellani, Gli studi spallanzaniani sulla digestione nei giornali e nelle dissertazioni, in Dynamis 3, 1983, p.91-147. 
Castellani, Quelques considérations sur les études de Spallanzani à propos de la respiration, in Physis 26, 1984, p. 533-546.

Castellani, Ricognizione di alcuni inediti spallanzaniani, con particolare riguardo agli studi sui pipistrelli (1793-1794). La «Memoria sul volo cieco dei pipistrelli dedicata al Senebier nelle due versioni», in Physis 26, 1984, p.433-483.

Cuvier, Georges, Eloges historiques de Charles Bonnet et H. B. de Saussure, in Recueil des éloges historiques lus dans les séances publiques de l'Institut Royal de France, Strasbourg et Paris, 1819, t. 2, p. 383-430.

Di Pietro, Pericle, Lazzaro Spallanzani, Modena, Aedes Muratoriana, 1979.

Di Pietro (édit.), Edizione nazionale delle opere di Lazzaro Spallanzani. Parte prima: Carteggi. Volume I-X, Modena, Mucchi, 1984-1988, 10 volumes, - En particulier vol. II (Carteggio con Charles Bonnet, 1984) et vol. VIII ( Carteggio con Jean Senebier, 1987).

Duchesneau, François, La physiologie des lumières. Empirisme, modèles et théories, La Haye, Martinus Nijhoff, 1982.

Gautier, Léon, La médecine à Genève jusqu'à la fin du dix-huitième siècle, Genève, Jullien, 1906.

Grmek, Mirko D., La théorie et la pratique de l'expérimentation biologique au temps de Spallanzani, in G. Montalenti et P. Rossi, Lazzaro Spallanzani e la biologia del Settecento, Firenze, Olschki, 1982, p. 321-352.

Grondona, Felice, Des expériments de Lazzaro Spallanzani sur la digestion aux applications thérapeutiques du suc gastrique, in Clio Med. 8, 1973, p. 285-294.

Leclercq, René, Histoire et avenir de la méthode expérimentale, Paris, Masson, 1960.

Lenoir, T.\&Elkana, Y. (edit.), Practice, raison, context: the dialogue between theory and experiment, in Science in Context 2, 1989, p. 3-212.

Manzini, Paola, Catalogo dei manoscritti di Lazzaro Spallanzani, Reggio Emilia, Comune, 1981.

Marx, Jacques, L'art d'observer au XVIII' siècle : Jean Senebier et Charles Bonnet, in Janus 61, 1974, p. 201-220.

Marx, Charles Bonnet contre les Lumières, Oxford, Voltaire Foundation, 1976.

Maunoir, Jean-Pierre, Eloge historique de M. Jean Senebier, Paris et Genève, Paschoud, 1810.

Mazzolini, Renato G.\& Roe, Shirley A., Science against the unbelievers: the correspondence of Bonnet and Needham, 1760-1780, Oxford, Voltaire Foundation, 1986.

Menuret de Chambaud, J.-J., Observation, in D. Diderot et J. L. d'Alembert, Encyclopédie, ou Dictionnaire raisonné des sciences, des arts et des métiers (2 éd.), Genève, 1777-79, p. 294-296.

Moeschlin-Krieg, Beate, Zur Geschichte der Regenerationsforschung im 18. Jahrhundert, Basel, Schwabe, 1953.

Montadon, Cléopâtre, Le développement de la science à Genève aux XVIII et XIX ${ }^{\circ}$ siècles, Vevey, Editions Delta, 1975.

Montalenti, Giuseppe \& Rossi, Paolo (réd.), Lazzaro Spallanzani e la biologia del Settecento. Teorie, esperimenti, istituzioni scientifiche, Firenze, Olschki, 1982.

Pilet, Paul-Emile, Jean Senebier, un des précurseurs de Claude Bernard, in Arch. Int. Hist. Sci. 15, 1962, p. 303-313.

Revilliod, Pierre, Physiciens et naturalistes genevois, Genève, Kundig, 1942.

Roe, Shirley A. (réd.), The natural philosophy of Albrecht von Haller, New York, Arno Press, 1981.

Roger, Jacques, Les sciences de la vie dans la pensée française du XVIII siècle. 2e éd., Paris, Armand Colin, 1971.

Rostand, Jean, Les origines de la biologie expérimentale et l'abbé Spallanzani, Paris, Fasquelle, 1951. 
Rostand, Les expériences de l'abbé Spallanzani sur la génération animale, in Arch. Int. Hist. Sci. 4, 1951, p. 413-447.

Rostand, Un grand biologiste: Charles Bonnet, expérimentateur et théoricien, Paris, Palais de la Découverte, 1966.

Rudolph, Gerhard, Les débuts de la transplantation expérimentale. Considérations de Charles Bonnet (1720-1793) sur la «greffe animale», in Gesnerus 34, 1977, p. 50-68.

Savioz, Raymond, Mémoires autobiographiques de Charles Bonnet de Genève, Paris, Vrin, 1948.

Savioz, La philosophie de Charles Bonnet de Genève, Paris, Vrin, 1948.

Sayous, André Edouard, La haute bourgeoisie de Genève et ses travaux scientifiques, in Rev. Hist. Suisse 2, 1940, p. 195-227.

Senebier, Jean, Qu'est-ce qui est requis dans l'Art d'observer?, in Verhandelingen Holl. Maatsch. Weetensch. Harlem. 13, 1772, 1-170.

Senebier, L'Art d'observer, Genève, Philibert et Chirol, 1775, 2 vols.

Senebier, Die Kunst zu beobachten. Aus dem Französischen übersetzt von Johann Friedrich Gmelin, Leipzig, 1776.

Senebier, Des considérations sur la méthode (de Spallanzani) de faire des expériences et les conséquences pratiques qu'on peut tirer en médecine de ses découvertes, in L. Spallanzani, Expériences sur la digestion de l'homme et de différentes espèces d'animaux, Genève, 1783, p. ICXLIX.

Senebier, Observations importantes sur l'usage du suc gastrique dans la chirurgie, Genève, 1785.

Senebier, Considérations sur la méthode suivie par Monsieur l'Abbé Spallanzani dans ses expériences sur la digestion, in L. Spallanzani, Opuscules de physique animale et végétale, Pavie, 1787, t. II, p. 313-350.

Senebier, Expériences sur l'action de la lumière solaire dans la végétation, Genève, 1788.

Senebier, Notice historique sur Lazare Spallanzani, Genève, Paschoud, 1800.

Senebier, Essai sur l'art d'observer et de faire des expériences. Seconde édition considérablement changée et augmentée, Genève, Paschoud, 1802, 3 vols.

Sonntag, Otto, The correspondence between Albrecht von Haller and Charles Bonnet, Bern, Huber, 1983.

Spallanzani, Lazzaro, Dissertazioni di fisica animale e vegetale. Aggiuntevi due lettere relative ad esse dissertazioni del celebre sig. Bonnet di Ginevra scritte all'autore, Modena, Società tipografica, 1780,2 vols.

Spallanzani, Expériences pour servir à l'histoire de la génération des animaux et des plantes. Traduit par Jean Senebier, Genève, Chirol, 1785.

Spallanzani, Opuscules de physique animale et végétale. Traduits par Jean Senebier, Pavie et Paris, Duplain, 1787, 2 vols.

Spallanzani, Mémoires sur la respiration. Traduits en français d'après son manuscrit inédit par Jean Senebier, Genève, Paschoud, 1803.

Spallanzani, I giornali delle sperienze e osservazioni relativi alla fisiologia della generazione e all embriologia sperimentale. Manoscritti inediti a cura di Castellani C. e Leone V.G., Milano, Episteme, 1978.

Spallanzani, Opere scelte. A cura di Castellani Carlo, Torino, UTET, 1978.

Spallanzani, Mariafranca, Nuovi studi su Spallanzani e la biologia del '700, in Quaderni Storici 16, 1981, p. 1080-1090. 
Starobinski, Jean, L'Essai de psychologie de Charles Bonnet; une version corrigée inédite, in Gesnerus 32, 1975, p.1-15.

Théodoridès, Jean, Lazzaro Spallanzani et le sens de l'orientation chez les chauves-souris, in Belloni Luigi, Per la storia della neurologia italiana, Milano, Istituto di Storia della Medicina, 1963, 65-92.

Tourdes, Joseph, Notices sur la vie littéraire de Spallanzani, in Spallanzani L., Expériences sur la circulation, Paris, Maradan, 1800, 1-112.

Trembley, Jean, Mémoire historique sur la vie et les écrits de Monsieur Abraham Trembley, Neuchâtel, 1787.

Trembley, Mémoires pour servir à l'histoire de la vie et de l'œuvre de M. Charles Bonnet, Berne, 1794.

Zimmermann, Johann Georg, Traité de l'expérience en général et en particulier dans l'art de guérir. Traduit de l'allemand par Lefèbvre de V., Paris, Vincent, 1774, 2 vols. 


\section{Summary}

\section{The contribution of Spallanzani's Geneva correspondents to the methodology of experimentation}

Certain traits of Spallanzanis work correspond to the principal values of the upper middle-class of Geneva of his time. Spallanzani was particularly well understood by Charles Bonnet, Jean Senebier and Abraham Trembley. Thus, the correspondence between these scholars is a very rich source for the reconstruction of the epistemological bases of the methodology of biological experimentation in the second half of the 18th century. The «Essai sur l'art d'observer et de faire des expériences» written by Senebier the parson is a masterly analysis and a kind of long sermon in favour of a method of investigation which Spallanzani the abbot preached exclusively by example.

\section{Zusammenfassung}

\section{Der Beitrag der Genfer Korrespondenten Spallanzanis zur Methodologie des Experimentes}

Gewisse Züge von Spallanzanis Werk entsprechen den wichtigsten Werten des Genfer Grossbürgertums seiner Zeit, der zweiten Hälfte des 18. Jahrhunderts. Bei Charles Bonnet, Jean Senebier und Abraham Trembley fand der italienische Naturforscher am meisten Verständnis. Der Briefwechsel dieser Gelehrten ist eine besonders reiche Quelle für die Rekonstruktion der epistemologischen Grundlagen der Methodologie des biologischen Experimentes in jener Zeit. Der «Essai sur l'art d'observer et de faire des expériences» des Pastors Senebier ist eine magistrale Analyse und gleichsam eine überzeugende Predigt zugunsten einer Forschungsmethode, die der Abbate Spallanzani nur durch sein Beispiel lehrte.

Professeur Mirko D. Grmek

10, rue de Savoie

F-75006 Paris 